\title{
Uma Arquitetura Multiagente para Monitoramento das Atividades de Tutoria em AVAs
}

\author{
Laysa Mabel de Oliveira Fontes ${ }^{1}$, Ricardo Alexsandro de Medeiros Valentim ${ }^{1}$, \\ Francisco Milton Mendes Neto ${ }^{2}$, Rafael Castro de Souza ${ }^{2}$ \\ ${ }^{1}$ Programa de Pós-Graduação em Engenharia Elétrica e de Computação - UFRN \\ Campus Universitário - BR 101 - Lagoa Nova - Natal, RN - Brasil \\ ${ }^{2}$ Programa de Pós-Graduação em Ciência da Computação - UERN/UFERSA \\ Av. Francisco Mota, 572 - Bairro Costa e Silva - Mossoró, RN - Brasil \\ mabel.fontes@ufersa.edu.br, ricardo.valentim@ufrnet.br, frafaelcastro, \\ miltonmendes\} @ufersa.edu.br
}

Resumo. Atualmente, o Brasil vive um momento muito especial no âmbito da Educação a Distância (EaD). Os Ambientes Virtuais de Aprendizagem (AVAs) têm contribuído para o crescimento e popularização da EaD. $O$ gerenciamento dos processos de aprendizagem em AVAs, com qualidade de interação e de acompanhamento, pode ser apontado como um dos potenciais indicadores de sucesso desse processo. No entanto, nem sempre essas atividades são desempenhadas adequadamente. Frente a este cenário, este trabalho tem como objetivo propor uma arquitetura multiagente para acompanhamento das atividades de tutoria em AVAs, baseada em informações extraídas por processos de Learning Analytics (LA), buscando promover a participação efetiva dos alunos.

\begin{abstract}
In regard to distance learning, Brazil currently lives a very special moment. Virtual Learning Environments (VLEs) have contributed to both the growth and the popularization of distance learning. The level of management of learning processes provided by VLEs, with great interaction and tracking quality, may be singled out as a key indicator of the success of this process. However, these activities aren't always performed adequately. Facing this scenario, this paper has the goal of proposing a multi-agent architecture for monitoring tutoring activities in VLEs, based on information extracted by Learning Analytics (LA) processes, in order to promote the effective participation of students.
\end{abstract}

\section{Introdução}

A Educação a Distância $(\mathrm{EaD})$ é uma modalidade de ensino e aprendizagem que tem crescido nos últimos anos no Brasil. De acordo com o Instituto Nacional de Estudos e Pesquisas Educacionais Anísio Teixeira (INEP), em 2014, foram registradas 1.341.842 matrículas em cursos de graduação a distância, o que representa uma participação de $17,1 \%$ do total de matrículas da educação superior [INEP 2015]. Ainda segundo o INEP, em 2014, o número de ingressos nos cursos a distância cresceu 41,2\% em comparação a 2013 [INEP 2015]. Os Ambientes Virtuais de Aprendizagem (AVAs) têm contribuído para o crescimento e popularização da EaD [Santana et al. 2014]. 
O gerenciamento dos processos de aprendizagem em AVAs, com qualidade de interação e de acompanhamento, pode ser apontado como um dos potenciais indicadores de sucesso desse processo. $\mathrm{Na} \mathrm{EaD}$ existe um ator responsável por essas atividades. Vale ressaltar que a nomenclatura utilizada e os papéis desempenhados pelos atores do processo de EaD podem apresentar variações nas instituições educacionais. Neste trabalho, o ator responsável pelo acompanhamento da aprendizagem do aluno em AVAs será chamado de tutor. De acordo com Preti (2005) e Bentes (2009), o tutor é um dos maiores responsáveis pelo sucesso dos cursos dessa modalidade de ensino, pois suas atribuições envolvem tarefas essenciais, como, por exemplo, orientação, condução e supervisão do processo de aprendizagem dos alunos.

Sabe-se que os dados gerados nas interações que os tutores têm com os alunos e com os recursos educacionais, disponíveis nos AVAs, são volumosos e pouco explorados. A área de Learning Analytics (LA) [Siemens et al. 2011] pode contribuir na descoberta de conhecimento potencialmente útil a partir desses dados. A utilização de Sistemas Multiagente (SMAs) também pode auxiliar em tarefas mais complexas dentro de um AVA, como, por exemplo, monitoramento de atividades de estudantes, captura de informações sobre o seu contexto dinâmico, recomendação de conteúdo baseada em seu perfil de usuário, dentre outras [Frade 2015]. No entanto, pouco tem sido pesquisado sobre as atividades de tutoria.

Frente a este cenário, este trabalho tem como objetivo propor uma arquitetura multiagente para acompanhamento das atividades de tutoria em AVAs, baseada em informações extraídas por processos de LA, buscando promover a participação efetiva dos alunos na modalidade a distância. Vale ressaltar que o desenvolvimento desta proposta está em andamento. Portanto, o intuito deste trabalho é apresentar o contexto geral desta proposta, enfatizando as etapas que já foram concluídas e os próximos passos de seu desenvolvimento.

Este artigo está organizado da seguinte forma: a Seção 2 descreve os conceitos do campo da LA; a Seção 3 apresenta os conceitos relacionados aos SMAs; a Seção 4 apresenta alguns trabalhos relacionados à temática desta proposta; na Seção 5, é descrita a metodologia utilizada para delimitação do escopo desta pesquisa; a Seção 6 apresenta a solução proposta e os resultados preliminares; e, por fim, a Seção 7 apresenta as considerações finais e os trabalhos futuros.

\section{Learning Analytics}

O campo de pesquisa denominado Learning Analytics (LA) pode ser definido como sendo a medição, coleta, análise e comunicação de dados sobre os alunos e os seus contextos, para fins de compreensão e otimização da aprendizagem nos ambientes em que esse processo ocorre [Siemens et al. 2011].

De acordo com Chatti et al. (2012), o processo da LA é basicamente dividido em três etapas distintas:

- Coleta de dados e pré-processamento: é responsável pela coleta dos dados que serão analisados e pelo pré-processamento, que consiste em uma conversão dos dados para um formato que possa ser utilizado pelas técnicas da LA; 
V Congresso Brasileiro de Informática na Educação (CBIE 2016)

Anais dos Workshops do V Congresso Brasileiro de Informática na Educação (CBIE 2016)

- Análises e ações: com base nos dados coletados e pré-processados, objetiva explorar os dados, de modo que se possa extrair informações a partir deles e planejar ações que possam ser tomadas a partir das informações obtidas, como, por exemplo, o monitoramento, a intervenção, a predição, etc.;

- Pós-processamento: esse estágio foca na melhoria contínua do processo da LA. Essa melhoria pode ser feita por meio da compilação de novos dados, através da adição de novos atributos, de modo que novos indicadores e análises possam ser obtidos ou então melhorar os já existentes.

Vale ressaltar que cada etapa do ciclo de vida gera uma saída para a etapa subsequente. Portanto, pode-se considerar que o processo global da LA é frequentemente um ciclo de vida iterativo [Chatti et al. 2012].

\section{Sistemas Multiagente}

Segundo Henderson-Sellers e Giorgini (2005), um Sistema Multiagente (SMA) é um sistema composto de agentes cooperativos ou competitivos que interagem entre si para atingir um objetivo comum ou individual.

É comum utilizar-se de SMAs para transformar grandes problemas em problemas menores e possibilitar, dessa forma, que cada agente possa utilizar sua habilidade para tratar esses pequenos problemas, modularizando o sistema e tornando mais fácil, por consequência, adicionar novas funcionalidades através da inclusão de novos agentes [Artero 2009].

Os SMAs podem ser organizados de diversas maneiras, sendo três os tipos mais comuns [Artero 2009]:

- Hierárquica: existe um agente superior que controla e toma as decisões, comunicando sua decisão para os demais agentes, que pertencem a um nível inferior na hierarquia;

- Comunidade de especialistas: todos os agentes estão em um mesmo nível, tendo cada agente uma especialidade em certo domínio. Nesse tipo de organização, a interação entre os agentes ocorre de acordo com regras previamente estabelecidas;

- Comunidade científica: os problemas são solucionados localmente e, em seguida, essas soluções são testadas e refinadas por agentes solucionadores de problemas.

A organização do SMA proposto neste trabalho será do tipo comunidade de especialistas, pois cada um dos agentes estará no mesmo nível, sendo cada um deles especialista em determinada tarefa.

\section{Trabalhos Relacionados}

Os campos de pesquisa de LA e SMAs têm sido amplamente utilizados no âmbito da educação a distância. Rabelo et al. (2015) apresentaram uma arquitetura para a análise de dados gerados pelos aprendizes durante um curso. Essa arquitetura foi desenvolvida a partir de tecnologias de Big Data, ontologias e padrões arquiteturais. Segundo os 
autores, essa plataforma apresentou bons resultados a partir da análise de 250.000 declarativas em um curso na modalidade blended learning com 60 inscritos.

Lima e Netto (2015) apresentaram uma análise dos resultados obtidos pelo sistema ARSEAVA, que tem como objetivo identificar e caracterizar as interações entre os estudantes de um determinado curso a distância e disponibilizar essas informações ao mediador do curso sob a forma de sociogramas e de tabelas. Segundo os autores, a análise realizada confirmou que as representações do sistema auxiliaram o mediador no processo pedagógico.

Amaral et al. (2015) propuseram a utilização da técnica de regressão linear para estimar o nível de interações dos alunos com base em variáveis comportamentais dos tutores. Segundo os autores, os resultados mostraram que é possível usar a técnica de regressão linear, a fim de obter inferências com boas taxas de precisão no que diz respeito à relação entre ações dos tutores no AVA e o aumento das interações dos alunos.

Romo, Guilherme e Queiroz (2013) construíram uma arquitetura multiagente para gestão de conteúdos educacionais. Como parte da arquitetura, foi especificado um conjunto de ontologias e agentes inteligentes, responsáveis por recuperar e integrar conteúdos educacionais, para as atividades de busca, autoria de metadados de Objetos de Aprendizagem (OAs) e autoria de cursos. Foi desenvolvido um SMA responsável por apoiar os usuários na autoria de cursos, implementando mecanismos para guiar os usuários na criação do curso, na anotação semântica do curso e na recomendação de OAs a serem utilizados no curso. O projeto foi aplicado para auxiliar professores na construção do curso de Matemática do nono ano do Ensino Fundamental.

Embora a presente proposta esteja nos mesmos campos de pesquisa que os trabalhos supracitados, esta apresenta uma perspectiva diferente. Este trabalho apresenta uma abordagem híbrida de LA e SMA para a identificação e acompanhamento das ações dos tutores que possam impactar na participação efetiva dos alunos na modalidade a distância. A arquitetura proposta é composta por: (i) um modelo de conhecimento; (ii) um sistema multiagente; e (iii) um módulo de avaliação de tutores e turmas.

\section{Material e Métodos}

Este trabalho foi empreendido por meio de uma pesquisa exploratória, que, por meio de levantamentos bibliográficos e análises de dados reais, estimulou o processo de compreensão do problema.

Em seguida, foram selecionadas as metodologias para a delimitação dos objetos de estudo. Para a aplicação da LA, foi selecionada a metodologia proposta em [Greller e Drachsler 2012] e para a modelagem do SMA, será utilizada a metodologia MASCommonKADS+ [Morais II 2010].

Para validar este trabalho, será realizado um estudo de caso na Secretaria de Educação a Distância (SEDIS) pertencente à Universidade Federal do Rio Grande do Norte (UFRN). Essa validação seguirá as orientações de uma pesquisa quantitativa, que, através da aplicação de um questionário, buscará apurar as opiniões dos atores envolvidos nesse processo, como forma de validar a eficácia da solução proposta em um cenário real. 
V Congresso Brasileiro de Informática na Educação (CBIE 2016)

Anais dos Workshops do V Congresso Brasileiro de Informática na Educação (CBIE 2016)

\section{Arquitetura e Resultados Preliminares}

Como já mencionado anteriormente, o intuito deste trabalho é apresentar o contexto geral da proposta de criação de uma arquitetura multiagente para acompanhamento das atividades de tutoria em AVAs, enfatizando as etapas que já foram concluídas e os próximos passos de seu desenvolvimento. A Figura 1 ilustra a arquitetura proposta neste trabalho.

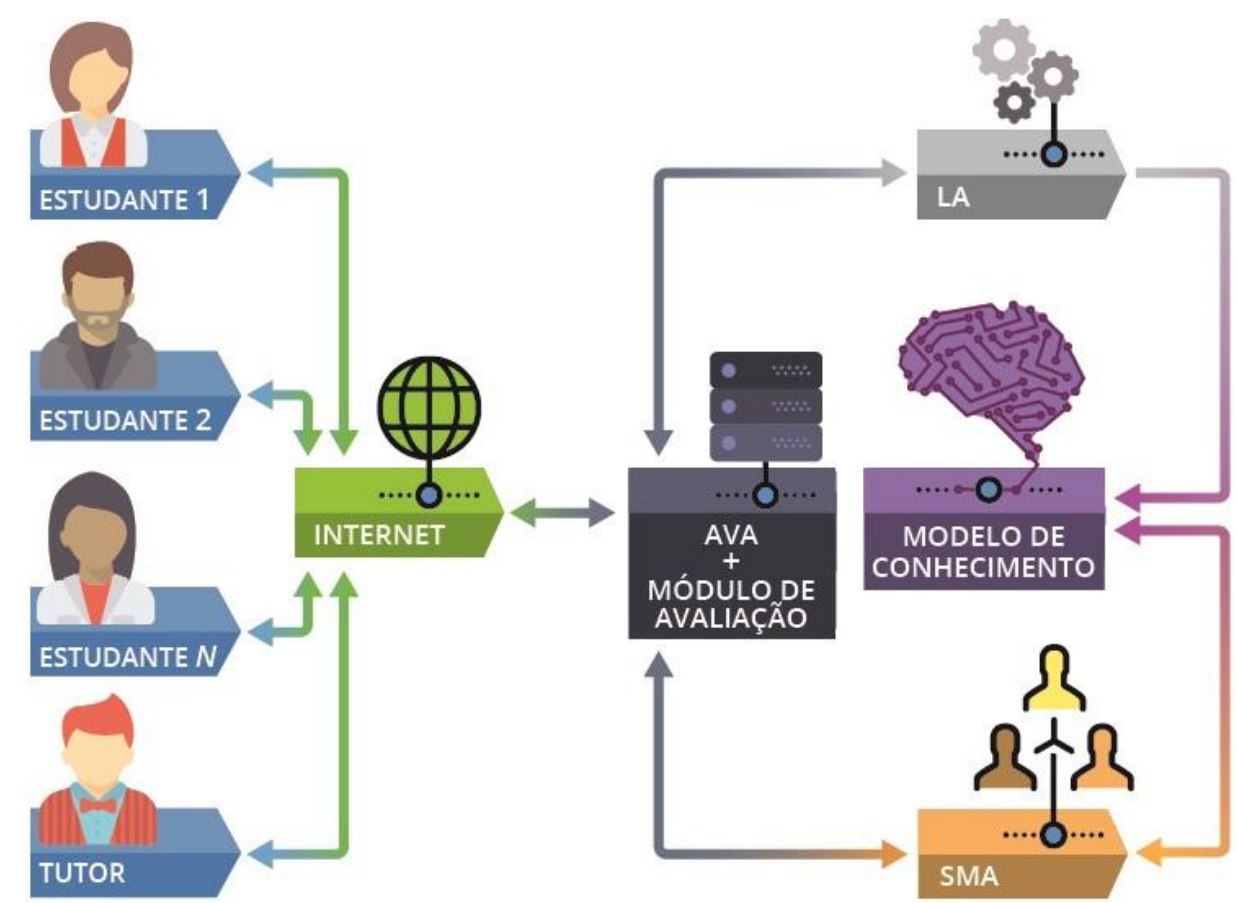

Figura 1. Arquitetura proposta

O desenvolvimento da arquitetura ilustrada na Figura 1 compreende quatro etapas: (i) criação do modelo de conhecimento; (ii) implementação do módulo de avaliação; (iii) modelagem e implementação do SMA; e (iv) validação da solução. Essas etapas serão descritas nas subseções a seguir.

Vale ressaltar que os dados utilizados nas etapas de desenvolvimento do modelo de conhecimento (Subseção 6.1) e do módulo de avaliação (Subseção 6.2) foram cedidos pela Secretaria de Educação a Distância (SEDIS) da Universidade Federal do Rio Grande do Norte (UFRN). Essa instituição usa o Moodle como AVA. Portanto, todas as informações que compõem o dataset, utilizado nas duas etapas citadas anteriormente, foram extraídas desse AVA.

Ao todo, foram retornados dados de 62 turmas, pertencentes a 10 cursos de graduação, que são: (i) Bacharelado em Administração Pública; (ii) Licenciatura em Ciências Biológicas; (iii) Licenciatura em Educação Física; (iv) Licenciatura em Física; (v) Licenciatura em Geografia; (vi) Licenciatura em Letras; (vii) Licenciatura em Matemática; (viii) Licenciatura em Pedagogia; (ix) Licenciatura em Química; e (x) Licenciatura em História. A base de dados histórica cedida corresponde aos anos de 2012 a 2013. No total, o dataset foi composto por informações de 2.227 alunos e 38 tutores. 
V Congresso Brasileiro de Informática na Educação (CBIE 2016)

Anais dos Workshops do V Congresso Brasileiro de Informática na Educação (CBIE 2016)

\subsection{Modelo de Conhecimento}

Para a criação do modelo de conhecimento, foi necessário, inicialmente, criar uma ferramenta para analisar, através da aplicação da LA, fatores comportamentais dos tutores que pudessem, ou não, estar associados com a participação efetiva dos alunos.

Essa ferramenta dispõe de basicamente dois módulos. O primeiro módulo é responsável pela extração das informações da base de dados e pela normalização dos dados. O segundo módulo é responsável pela aplicação da LA propriamente dita.

Os atributos dos tutores analisados pela ferramenta foram: (i) número de questionários criados; (ii) número de tópicos criados nos fóruns; (iii) média de postagens em tópicos dos fóruns; (iv) taxa de visualização em fóruns; (v) taxa de visualização em tópicos dos fóruns; (vi) número de tarefas criadas; (vii) número de tarefas avaliadas; (viii) média de postagens em chats; (ix) total de cliques; (x) número de páginas criadas; (xi) número de URLs criadas; e (xii) número de arquivos criados.

Quanto aos comportamentos das turmas, foram analisados os seguintes atributos: (i) taxa de participação nos questionários; (ii) tempo médio para finalização dos questionários; (iii) média de tópicos criados nos fóruns; (iv) média de postagens em tópicos dos fóruns; (v) média de acesso em fóruns; (vi) média de acesso em tópicos dos fóruns; (vii) taxa de submissão de tarefas; (viii) média de postagens em chats; (ix) média de cliques; (x) média de páginas visualizadas; e (xi) média de arquivos visualizados.

Primeiramente, a ferramenta extrai as informações dos cursos cadastrados, dos tutores de cada curso e dos alunos matriculados em cada curso. Após a obtenção dessas informações, é iniciado o procedimento de extração das informações relacionadas aos comportamentos a serem analisados.

Após a extração dessas informações, o primeiro módulo as armazenam em um arquivo no formato .arff [WEKA 2016], que servirá como arquivo de entrada para o segundo módulo, que é responsável pelo processamento dessas informações.

Para identificar a correlação dos comportamentos dos tutores em relação ao comportamento da turma, foi utilizado o coeficiente de correlação de Pearson [Sharma 2012], e para o nível de significância da análise, foi utilizado o valor para $\alpha=0,05$ [Rubin 2012]. Portanto, o nível de confiança dos resultados é de 95\% [Rubin 2012]. Também foi utilizada a técnica Z-Score [Warner 2012] para remover os outliers ${ }^{1}$.

Ao todo foram estudados doze comportamentos dos tutores e onze comportamentos das turmas, gerando uma combinação total de cento e trinta e duas correlações, das quais cento e cinco correlações não passaram no teste de confiança, ou seja, a indicação foi de aceitação da hipótese nula, e vinte e sete correlações passaram no teste de confiança, sendo indicada a aceitação da hipótese alternativa. Das vinte e sete correlações, três apresentaram correlação forte positiva, quinze apresentaram correlação moderada positiva e nove apresentaram correlação fraca positiva. Em [Souza et al. 2016a], é apresentado um estudo detalhado com parte dos comportamentos analisados neste trabalho.

\footnotetext{
${ }^{1}$ Outliers são valores atípicos, isto é, uma observação que apresenta um valor muito afastado em relação aos demais valores [Hawkins 2013].
} 
A partir das vinte e sete correlações que passaram no teste de confiança, será possível criar o modelo de conhecimento. Portanto, para concluir esta etapa, pretende-se criar uma ontologia para representar o modelo de conhecimento.

\subsection{Módulo de Avaliação}

Durante o desenvolvimento deste trabalho, foi percebida a necessidade de criação de um módulo capaz de avaliar as atividades dos tutores, bem como de suas respectivas turmas, a fim de permitir que os próprios tutores, professores e coordenadores de curso pudessem acompanhar estas turmas, em tempo real, de modo que situações de baixa participação pudessem ser atenuadas. Portanto, esse módulo objetiva facilitar a compreensão e interpretação dos dados gerados pelas avaliações e sugerir estratégias pedagógicas quando problemas de baixa participação forem detectados.

O módulo foi construído utilizando a linguagem de programação Java [Oracle 2016a]. Para a classificação dos dados, foi utilizado o algoritmo K-Means [Macqueen 1967], disponível na biblioteca WEKA. E para a construção do Core III (detalhado mais adiante), foi utilizada a linguagem de programação JavaServer Faces [Oracle 2016b], em conjunto com a API PrimeFaces [PrimeFaces 2016].

A arquitetura do módulo proposto contém três núcleos (cores) de processamento de dados: (i) núcleo de extração de dados (Core I); (ii) núcleo de processamento de dados (Core II); e (iii) núcleo de apresentação de dados (Core III).

O Core I é responsável pela extração das informações no banco de dados. Este núcleo extrai os mesmos atributos dos tutores e das turmas discutidos na Subseção 6.1. Após a extração dos dados, este núcleo armazena os dados no formato .arff. O dataset gerado pelo Core I servirá como entrada de dados para o Core II.

O Core II tem o objetivo de processar as informações que foram extraídas pelo Core I. São realizados dois tipos de processamento no Core II: (i) criação e treinamento do algoritmo K-Means; e (ii) cálculo da posição relativa dos atributos analisados.

A criação e treinamento do algoritmo de classificação consistem em criar, para cada atributo analisado, a sua própria rede de classificação. Em outras palavras, cada atributo do tutor e da turma, que será analisado, terá sua própria rede de classificação, que será classificada por meio da técnica K-Means, utilizando-se do critério da distância euclidiana entre os centróides, para $k=3$, ou seja, serão criados três agrupamentos. A partir disso, será possível identificar se o atributo em questão pertence à categoria dos que tiveram baixo desempenho, desempenho moderado ou um bom desempenho. Vale ressaltar que essa rede de classificação será treinada por meio das instâncias do dataset resultante do processamento do Core I.

Além da classificação propriamente dita, que tem por objetivo classificar cada um dos atributos por meio da técnica de agrupamento, o Core II calcula também a posição relativa de cada atributo avaliado em comparação com os dados das demais turmas. Para isso, é utilizada a técnica de normalização Min-Max [Faceli et al. 2011].

Desse modo, além da classificação, que irá permitir uma avaliação de cada atributo do tutor e da turma, por meio da técnica de agrupamento de dados, será ainda exposto um gráfico informando a posição relativa do desempenho da turma em comparação com as demais turmas. 
O Core III é responsável pela exibição das informações processadas no Core II e das estratégias pedagógicas, e também por permitir a interação do usuário com o módulo. Resumidamente, após a escolha do curso a ser avaliado, os seus dados são extraídos em tempo real (Core I), e, em seguida, são processados no Core II, e, por fim, as respectivas informações de classificação e desempenho relativo são apresentadas (Core III). Vale ressaltar que o desenvolvimento deste módulo de avaliação já foi concluído e pode ser conferido em [Souza et al. 2016b].

\subsection{Sistema Multiagente}

O intuito do SMA proposto neste trabalho é operar no acompanhamento das atividades de tutoria no AVA Moodle, de modo autônomo e proativo. Para isto, será necessário traçar um plano de ação para o SMA, que irá executá-lo com base no modelo de conhecimento, detalhado na Subseção 6.1. Vale ressaltar que um dos objetivos do plano de ação é sugerir estratégias pedagógicas que poderão ser visualizadas na interface do módulo de avaliação, discutido da Subseção 6.2.

Esta etapa do desenvolvimento consiste na modelagem e implementação do SMA. Para isto, será necessário definir a quantidade de agentes que irão compor a arquitetura, de acordo com a quantidade de tarefas que serão definidas para solucionar o problema. Para a modelagem do SMA, será utilizada a metodologia MASCommonKADS+. E para a implementação do SMA, será utilizado o framework JADE [JADE 2016]. Vale ressaltar que a execução desta etapa será iniciada após a conclusão do processo de criação do modelo de conhecimento.

\subsection{Validação}

Por fim, a última etapa desta proposta consiste na validação da solução. Após finalizar todas as etapas discutidas nas subseções anteriores, será realizado um estudo de caso com algumas disciplinas ofertadas pela Secretaria de Educação a Distância da Universidade Federal do Rio Grande do Norte. Ao final do estudo de caso, será aplicado um questionário, onde será possível receber o feedback dos atores envolvidos nesse processo, como forma de validar a eficácia da solução proposta em um cenário real.

\section{Considerações Finais e Trabalhos Futuros}

Este trabalho apresentou um estudo preliminar sobre a criação de uma arquitetura multiagente para acompanhamento das atividades de tutoria em AVAs, buscando promover a participação efetiva dos alunos da modalidade a distância.

Espera-se que esta pesquisa possa: (i) proporcionar uma melhor compreensão sobre fatores comportamentais dos tutores que possam, ou não, impactar na participação efetiva dos alunos; (ii) permitir que, através do módulo de avaliação, os coordenadores de cursos, os professores e os tutores possam refletir sobre os desempenhos obtidos; e (iii) potencializar a atuação qualificada do tutor, fomentando o engajamento e a qualidade das aprendizagens dos alunos.

Como trabalhos futuros, pretende-se: (i) coletar mais dados relacionados aos comportamentos dos tutores e das turmas, a fim de identificar novas correlações; (ii) criar uma ontologia para representar o modelo de conhecimento; (iii) realizar novas 
V Congresso Brasileiro de Informática na Educação (CBIE 2016)

Anais dos Workshops do V Congresso Brasileiro de Informática na Educação (CBIE 2016)

análises com outros tipos de técnicas; (iv) modelar e implementar o SMA; e (v) realizar o estudo de caso para validar a eficácia da solução proposta.

\section{Referências}

Amaral, Y., Rodrigues, R. L., Soares, F., Gonçalves, A. F. D. e Maciel, A. M. A. (2015) "Analysis of the linearity relation between the tutor's actions and the learners' interactions in Distance Education courses". In: Anais do Simpósio Brasileiro de Informática na Educação - SBIE, 26, Maceió.

Artero, A. O. (2009), Inteligência Artificial - Teoria e Prática. $1^{a}$ Edição. São Paulo: Livraria da Física.

Bentes, R. F. (2009) "A Avaliação do Tutor". In: Litto, F. M. e Formiga, M. (org.). Educação a Distância: o estado da arte. 1. ed. São Paulo: Pearson Education do Brasil, p. 166-170, 2009.

Chatti, M. A., Dyckhoff, A. L., Schroeder, U. e Thüs, H. (2012). A Reference Model for Learning Analytics. In: International Journal of Technology Enhanced Learning, v. 4, n. 5/6, p. 318-331.

Faceli, K., Lorena, A. C., Gama, J. e Carvalho, A. C. P. L. F. (2011), Inteligência Artificial: Uma Abordagem de Aprendizado de Máquina. Rio de Janeiro: LTC.

Frade, R. V. C. (2015) "UNIVIRTUAL - Ambiente Virtual 3D Multiagente com Recomendação Personalizada de Objetos de Aprendizagem". 2015. 90 f. Dissertação (Mestrado em Ciência da Computação) - Universidade Estadual do Rio Grande do Norte, Universidade Federal Rural do Semi-Árido, Mossoró.

Greller, W. e Drachsler, H. (2012). Translating Learning into Numbers: a generic framework for learning analytics. Journal of Educational Technology \& Society, v. 15, n. 3 , p. $42-57$.

Hawkins, D. (2013), Identification of Outliers, Springer Science \& Business Media.

Henderson-Sellers, B. e Giorgini, P. (2005), Agent-Oriented Methodologies. Guernsey: IGI Global.

INEP - Instituto Nacional de Estudos e Pesquisas Educacionais Anísio Teixeira. (2015) "Censo da Educação Superior 2014 - Notas Estatísticas". Disponível em: $<$ http://download.inep.gov.br/educacao_superior/censo_superior/documentos/2015/n otas_sobre_o_censo_da_educacao_superior_2014.pdf>. Acesso em: 26 maio 2016.

JADE. (2016) "JAVA Agent DEvelopment Framework". Disponível em: $<$ http://jade.tilab.com/>. Acesso em: 26 maio 2016.

Lima, D. P. R. e Netto, J. F. M. (2015) "Análise dos Resultados de um Sistema Multiagente que Identifica e Caracteriza as Relações Sociais dos Alunos de um Ambiente Virtual de Aprendizagem". In: Anais do Simpósio Brasileiro de Informática na Educação - SBIE, 26, Maceió.

Macqueen, J. (1967) "Some Methods for Classification and Analysis of Multivariate Observations". In: Proceedings of the Fifth Berkeley Symposium on Mathematical Statistics and Probability. 
V Congresso Brasileiro de Informática na Educação (CBIE 2016)

Anais dos Workshops do V Congresso Brasileiro de Informática na Educação (CBIE 2016)

Morais II, M. J. D. O. (2010) "MAS-CommonKADS+: Uma Extensão à Metodologia Mas-CommonKADS para Suporte ao Processo Detalhado de Sistemas Multiagentes Racionais". Dissertação de Mestrado. Universidade Estadual do Ceará - UECE. Fortaleza.

Oracle. (2016a) "Software Java". Disponível em: $<$ http://www.oracle.com/br/java/overview/index.html >. Acesso em: 26 maio 2016.

Oracle. (2016b) "JavaServer Faces Technology". Disponível em: $<\mathrm{http}$ //www.oracle.com/technetwork/java/javaee/javaserverfaces-139869.html>. Acesso em: 26 maio 2016.

Preti, O. (2005) "Apoio à Aprendizagem: o orientador acadêmico". In: Almeida, M. E. B. e Moran, J. M. (Ed.). Integração das Tecnologias na Educação: salto para o futuro. Brasília: Ministério da Educação.

PrimeFaces. (2016) "Ultimate UI Framework for Java EE". Disponível em: $<$ http://www.primefaces.org/>. Acesso em: 26 maio 2016.

Rabelo, T., Lama, M., Amorin, R. e Vidal, J. C. (2015) "Plataforma de Serviços Inteligentes de Learning Analytics para grandes quantidades de dados". In: Anais dos Workshops do Congresso Brasileiro de Informática na Educação - CBIE, 4, Maceió.

Romo, B. S., Guilherme, I. R. e Queiroz, J. (2013) "Uma Arquitetura Multiagente para Sistemas Web Semântico para Gestão de Conteúdos Educacionais". In: Anais dos Workshops do Congresso Brasileiro de Informática na Educação, Campinas.

Rubin, A. (2012), Statistics for Evidence-Based Practice and Evaluation, Cengage Learning.

Santana, M. A., Santos Neto, B. F., Costa, E. B. e Silva, I. C. L. (2014) "Avaliando o Uso das Ferramentas Educacionais no Ambiente Virtual de Aprendizagem Moodle". In: Anais do Simpósio Brasileiro de Informática na Educação - SBIE, 25, Dourados.

Sharma, J.K. (2012), Business Statistics, Pearson Education India.

Siemens, G., Gasevic, D., Haythornthwaite, C., Dawson, S., Shum, S. B., Ferguson, R., Duval, E., Verbert, K. e Baker, R. S. J. D. (2011). Open Learning Analytics: an integrated \& modularized platform. In: Society for Learning Analytics Research.

Souza, R. C., Mendes Neto, F. M., Santos, A. M., Fontes, L. M. O. e Valentim, R. A. M. (2016a) "Investigação acerca do Impacto dos Comportamentos dos Tutores em Turmas de Ensino a Distância". In: WCBIE - Anais dos Workshops do CBIE 2016 WMDE, Uberlândia.

Souza, R. C., Mendes Neto, F. M., Santos, A. M., Fontes, L. M. O., Rodrigues, E. N. H. D. e Valentim, R. A. M. (2016b) "Um Ambiente Inteligente de Avaliação de Comportamentos de Tutores e Turmas no Ambiente Virtual de Aprendizagem Moodle". In: WCBIE - Anais dos Workshops do CBIE 2016 - WAvalia, Uberlândia.

Warner, R. M. (2012), Applied Statistics: From Bivariate Through Multivariate Techniques, SAGE.

WEKA - Waikato Environment for Knowledge Analysis. (2016) "ARFF". Disponível em: $<$ http://weka.wikispaces.com/ARFF>. Acesso em: 26 maio 2016. 